\title{
Moyamoya Disease: Treatment and Outcomes
}

\author{
Tackeun Kim, ${ }^{a, b}$ Chang Wan Oh, ${ }^{a, b}$ Jae Seung Bang, ${ }^{a, b}$ Jeong Eun Kim, ${ }^{b, c}$ Won-Sang Cho ${ }^{b, c}$ \\ ${ }^{a}$ Department of Neurosurgery, Seoul National University Bundang Hospital, Seongnam, Korea \\ ${ }^{b}$ Department of Neurosurgery, Seoul National University College of Medicine, Seoul, Korea \\ 'Department of Neurosurgery, Seoul National University Hospital, Seoul, Korea
}

\begin{abstract}
Although the pathogenesis of moyamoya disease (MMD) has not been fully elucidated, the effectiveness of surgical revascularization in preventing stroke has been addressed by many studies. The main mechanism of surgical revascularization is augmenting the intracranial blood flow using an external carotid system by either direct bypass or pial synangiosis. This can improve resting cerebral blood flow as well as vascular reserve capacity. For direct revascularization, the superficial temporal artery is used as the donor artery in most cases, although the occipital artery may be used in limited cases. Usually, the cortical branch of the middle cerebral artery is selected as the recipient of direct anastomosis. As for indirect revascularization, various techniques using different kinds of connective tissues have been introduced. In some cases, reinforcing the anterior cerebral artery and the posterior cerebral artery territories can be considered. The effectiveness of surgical revascularization for preventing ischemic stroke had been generally accepted by many studies. However, for preventing hemorrhagic stroke, new evidence has been added by a recent randomized controlled trial. The incidence of peri-operative complications such as stroke and hyperperfusion syndrome seems to be high due to the nature of the disease and technical demands for treatment. Preventing and adequately managing these complications are essential for ensuring the benefits of surgery.
\end{abstract}

Keywords Moyamoya disease; Cerebral revascularization; Treatment outcome

\author{
Correspondence: Chang Wan Oh \\ Department of Neurosurgery, Seoul \\ National University Bundang Hospital, \\ 82 Gumi-ro 173 Beon-gil, Bundang-gu, \\ Seongnam 13620, Korea \\ Tel: +82-31-787-7162 \\ Fax: +82-31-787-4059 \\ E-mail:wanoh@snu.ac.kr
}

Received: December 7, 2015

Revised: January 17, 2016

Accepted: January 17, 2016

The authors have no financial conflicts of interest.

\section{Introduction}

Moyamoya disease (MMD) is an idiopathic disease with a progressive nature leading to recurrent stroke due to occlusion of the terminal internal carotid arteries. ${ }^{1}$ Although a recent genetic study identified a possible susceptibility gene, ${ }^{2}$ the pathogenesis of MMD has not been fully defined. Nevertheless, the incidence and prevalence of MMD has gradually increased. ${ }^{3}$ Revascularization surgery for symptomatic MMD is considered the standard treatment for preventing further stroke. ${ }^{4,5}$ The main objective of surgery is to augment intracranial blood flow using an external carotid system by either direct bypass or pial synangiosis. Although this phenomenon is a natural adaption process for compensating for stenosis of the internal carotid artery (ICA), it can be achieved readily by either extracranial-intracranial bypass or vasculogenesis using indirect pial synangiosis for symptomatic patients. In particular, surgical revascularization to prevent ischemic stroke is an effective treatment for patients with MMD with an ischemic presentation. ${ }^{6}$ Although still controversial, revascularization surgery to prevent further hemorrhage has also been performed by many neurosurgeons. ${ }^{7}$ Recently, an endovascular treatment has also been introduced through embolization of a ruptured aneurysm of the collateral vessels and angioplasty of a narrowed segment with or without stenting., 


\section{Conservative treatment of MMD}

Long-term prognosis for patients with non-surgically treated MMD is not fully understood. However, some reports have described its natural clinical course and the results of conservative treatment. Kuroda et al. ${ }^{10}$ reported a disease progression rate of approximately $20 \%$ over 6 years. Being female was identified as an independent risk factor for disease progression by multivariate analysis. Other investigations on the progression rate of the unaffected side of surgically treated unilateral MMD reported that six of the 41 cases (14.6\%) exhibited contralateral progression during the mean follow-up of 34 months. ${ }^{11}$ Considering these reports, MMD seems to have a progressive nature. Among many studies examining risk factors for MMD progression, the presence of thyroid disease such as Graves' disease has been a well-known medical condition linked to rapid progression of MMD. ${ }^{12,13}$ Recently, the RNF213 variant was suggested as a possible causative genetic alteration leading to the development as well as progression of $\mathrm{MMD}^{14}$

Because surgical revascularization has been recommended for symptomatic patients with impaired hemodynamics, some studies have described the outcomes of conservative treatment among asymptomatic or hemodynamically stable patients with MMD. A multi-center, nationwide survey for conservative treatment results was conducted in 2007 in Japan. The authors reported the annual stroke rate as $3.2 \%$ from the observation of 34 asymptomatic patients conservatively followed over 44 months. Hemodynamic disturbance was revealed to be a risk factor for newly developed stroke. ${ }^{15}$ In a North American series, the rates of annual ischemic and hemorrhagic stroke rate were reported as $13.3 \%$ and $1.7 \%$, respectively. Being female and smoking were risk factors for stroke development. ${ }^{16} \mathrm{Cho}$ et al. reported an annual stroke rate of $4.5 \%$ among 241 hemodynamically stable patients with MMD over 83 months. The annual stroke rate was higher in the hemorrhagic presentation group (5.7\%) than the ischemic presentation group (4.2\%) or the asymptomatic group (3.4\%). They found familial disease and thyroid disease to be risk factors affecting stroke occurrence. ${ }^{17}$ As for ischemic presenting MMD, 5.6\% of the annual ischemic stroke rate also reported that posterior circulation involvement was a strong risk factor for ischemic stroke. ${ }^{18}$

Antiplatelet treatment for preventing stroke in patients with MMD had been utilized by many physicians, especially in nonAsian areas. According to the reports of a worldwide survey, $31 \%$ of responders agreed to use long-term acetylsalicylic acid. ${ }^{19}$ However, the evidence for antiplatelet treatment is lacking. Recently, the efficacy of antiplatelet therapy for preventing stroke was investigated in a cohort study with a large sample size. According to the authors, antiplatelet therapy could not prevent recurrent cerebral infarction for ischemic presenting patients with MMD. The nature of the ischemic insult in patients with MMD is not an embolic infarction, but instead is mainly a hemodynamic infarction. The pathologic changes of the MMD vessels near the ICA bifurcation are not a type of endothelial damage, which is prone to platelet adhesion. Therefore, theoretically, antiplatelet drugs will not be effective for preventing ischemic stroke in patients with MMD. Although antiplatelet users are subject to hemorrhagic complications, the therapy was not associated with an increase in cerebral hemorrhage among patients with $\mathrm{MMD}^{20}$ Thus, prescribing antiplatelet agents for symptomatic patients with MMD should not yet be considered as an alternative treatment.

\section{Indication of surgical revascularization for MMD}

The most important goal of surgical revascularization is to prevent cerebral infarction by improving cerebral blood flow $(\mathrm{CBF})$ and restoring reserve capacity. Generally accepted indications for revascularization include recurrent clinical symptoms due to 1) apparent cerebral ischemia or 2) decreased regional $\mathrm{CBF}$, vascular response, and reserve in perfusion studies. ${ }^{4}$ However, this cannot be strictly applied to all patients due to the different clinical course between pediatric and adult patients with MMD. Since pediatric MMD is characteristically more progressive than in adult patients, revascularization surgery is indicated in most children with MMD. ${ }^{21}$ As such, it is very important that early diagnosis and active intervention happen before irreversible brain damage occurs in order to achieve a favorable clinical outcome in children. ${ }^{22}$

As described earlier, the stroke incidence in asymptomatic patients with MMD or MMD with a relatively stable hemodynamic status seems to be significant. To determine a treatment strategy for these patients, several factors should be considered and shared with the patient. In the case of crescendo TIA, surgical treatment should be considered even if the hemodynamic status is nearly normal. Since the benefit-risk difference is narrow and mostly dependent on peri-operative complications, a critical consideration in selecting surgery is the complication profile of the surgeon and institute. ${ }^{6}$

\section{Statistics of surgical revascularization for MMD}

To our knowledge, national statistics regarding surgical treat- 


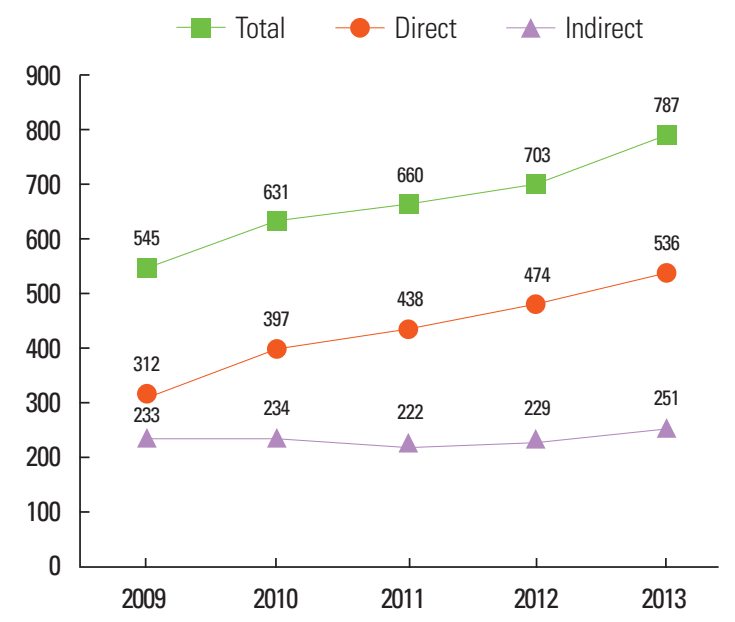

Figure 1. This line charts demonstrates the change in the number of revascularization surgeries from 2009 to 2013 in Korea. The number of indirect revascularization surgeries is constant over this period while the number of direct revascularization surgeries gradually increases.

ment for MMD have not been published. According to the database of the National Health Insurance Service, a universal insurer in Korea, 10,506 patients were newly diagnosed with MMD between 2009 and 2013. ${ }^{3}$ During the same period, 3,326 revascularization surgeries were performed in Korea. Among those surgical procedures, 2,157 (64.8\%) were direct (including combined) and 1,169 (35.1\%) were indirect surgery. As a crude calculation, this suggests that $31.4 \%$ of newly diagnosed patients with MMD were treated using surgical modalities. Throughout this period, the number of direct revascularization surgeries has gradually increased while the number of indirect revascularization surgeries has remained stagnant (Figure 1).

\section{Direct revascularization for MMD}

Since the 1970s, direct bypass has been used in patients with MMD. ${ }^{23}$ Following successful anastomosis between donor and recipient arteries, improvement in flow is achieved immediately after surgery. ${ }^{24}$ However, this procedure is technically difficult, requiring a highly trained surgeon. The vascular diameters of the superficial temporal artery (STA) and cortical arteries are important factors to determine the possibility of a direct bypass. In the advanced stage of MMD, most of the cortical arteries have shrunk to a small caliber and the vessel walls of patients with MMD tend to be more fragile. Post-operative hyperperfusion syndrome is another considerable problem leading to neurologic deterioration, which often develops after direct bypass surgery.

As a donor artery, the STA is selected in the majority of cases (Figure 2, black arrowheads). ${ }^{6,24-29}$ After harvesting the STA

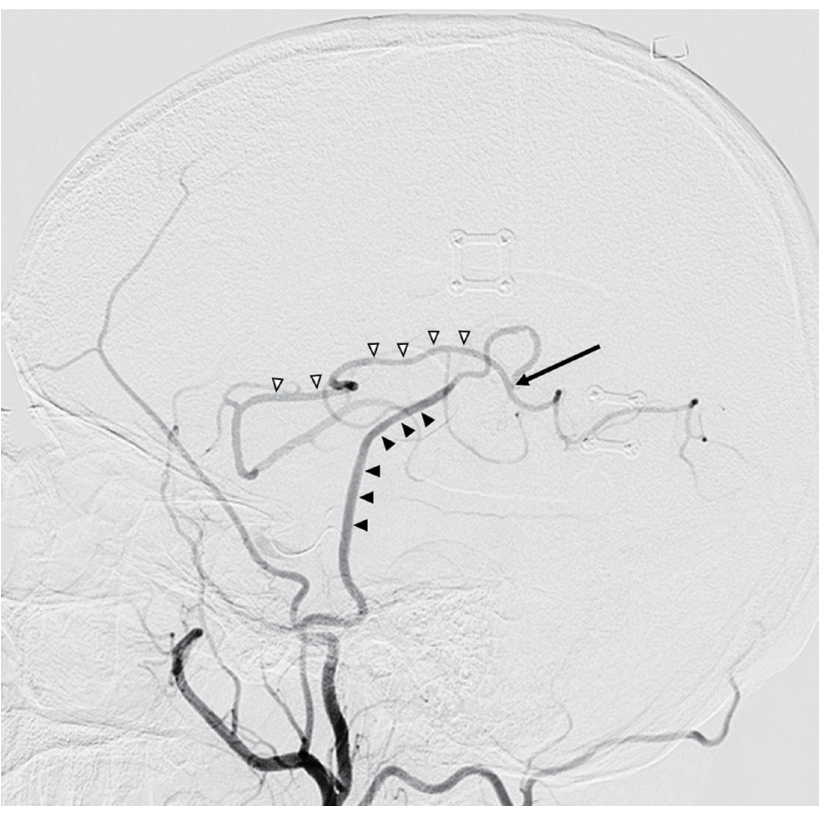

Figure 2. Post-operative angiography of the external carotid artery. Black arrowheads indicate the superficial temporal artery (donor artery). The tip of the black arrow is the anastomosis site to the angular artery (recipient artery). The middle cerebral artery is supplied from the superficial temporal artery with a reverse direction (white arrowheads).

from the scalp, papaverine is applied to the surface of the STA to prevent vasospasm. Various craniotomies can be performed according to the surgeon's preference and region of hypoperfusion. In addition, naturally formed trans-dural or trans-calvarial collateral channels should be preserved without injury. Most recipients are cortical branches of the middle cerebral artery (MCA, Figure 2, white arrowheads). The Chater's point is a useful landmark for identifying the larger cortical branches. To reinforce posterior cerebral artery (PCA) blood flow, the occipital artery $(\mathrm{OA})$ has been used as a donor for anastomosis to the PCA cortical branch. ${ }^{30}$ Of note, various indirect revascularization methods are combined in most cases.

The amount of blood flow through the bypass pedicle can be measured with an ultrasonic flow meter and visualized by indocyanine green angiography. ${ }^{31}$ During the follow-up period, digital subtraction with angiography and quantitative magnetic resonance angiography is useful to evaluate the patency and the amount of bypass flow (Figure 3).

\section{Indirect revascularization for MMD}

As described, direct bypass is a somewhat difficult procedure in young pediatric patients or adult patients with advanced MMD due to the small caliber of the recipient artery. In such cases, fortunately, indirect bypass using various connective tissues has been effective, possibly due to the nature of 
the disease in promoting spontaneous leptomeningeal collateral formation. ${ }^{32}$ The one of advantage of indirect revascularization is that it is relatively easier to perform than direct surgery. This makes the operation time shorter, which is important in preventing complications. In addition, post-operative hyperperfusion syndrome rarely develops after indirect revascularization. However, it takes more time to improve cerebral blood flow, because neovascularization from connective tissue

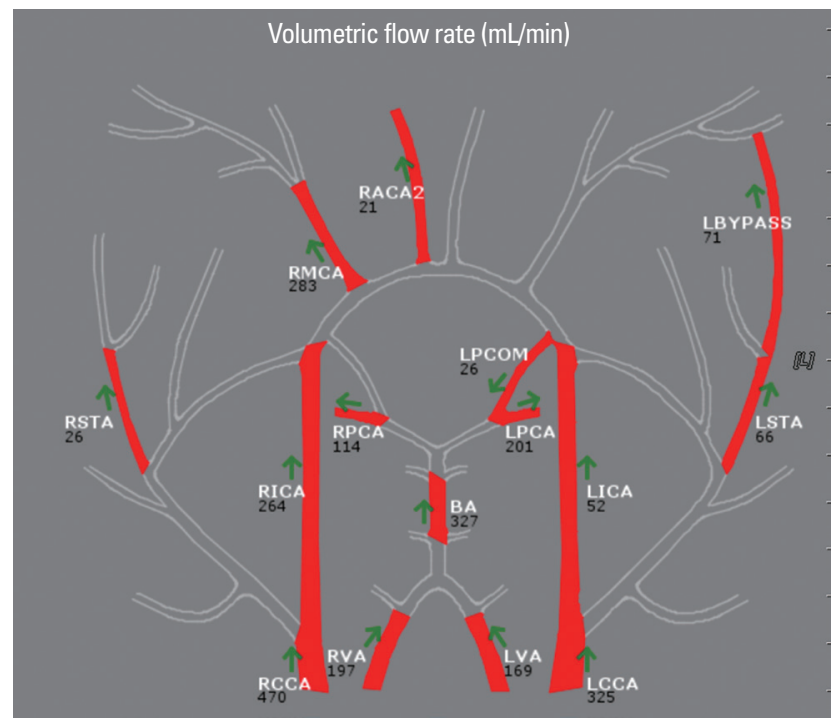

Figure 3. The quantitative magnetic resonance angiography shows intact bypass flow through the superficial temporal artery. is not immediate.

The surgical procedures can be classified according to the various tissues covering the brain. Historically, encephalomyo synangiosis (EMS) for MMD was introduced by Karasawa et al. in the 1970s. ${ }^{33}$ In this operation, the deep temporal artery (DTA), supplying the temporalis muscle, becomes the main supplier of neovascularization. The temporalis muscle or dissected inner fascia can be covered with the brain surface being sutured with dura. Of note, swollen muscle can cause brain compression after surgery.

In an encephalo-duro-arterio synangiosis (EDAS) operation, a skin incision is usually made along the parietal branch of the STA. After harvesting the STA with the surrounding galea and periosteum, craniotomy and durotomy are made under the STA flap with a galea cuff. Each margin of the dura and galea are sutured together to cover the brain with an arterial flap. Typically, the STA is connected to both the proximal and distal side (Figure 4).

Other indirect methods such as encephalo-myo-arterio synangiosis (EMAS), encephalo-duro-arterio-myo synangiosis (EDAMS), and encephalo-galeo synangiosis (EGS) are performed as variants of EMS and EDAS. ${ }^{33-38}$

Bi-frontal indirect bypass can be considered if the patient has frontal lobar hypoperfusion. ${ }^{39-42}$ In MMD, the diameter of the frontal branch of the STA becomes thin and branched at the midline. Thus, without a large artery, the galea and the
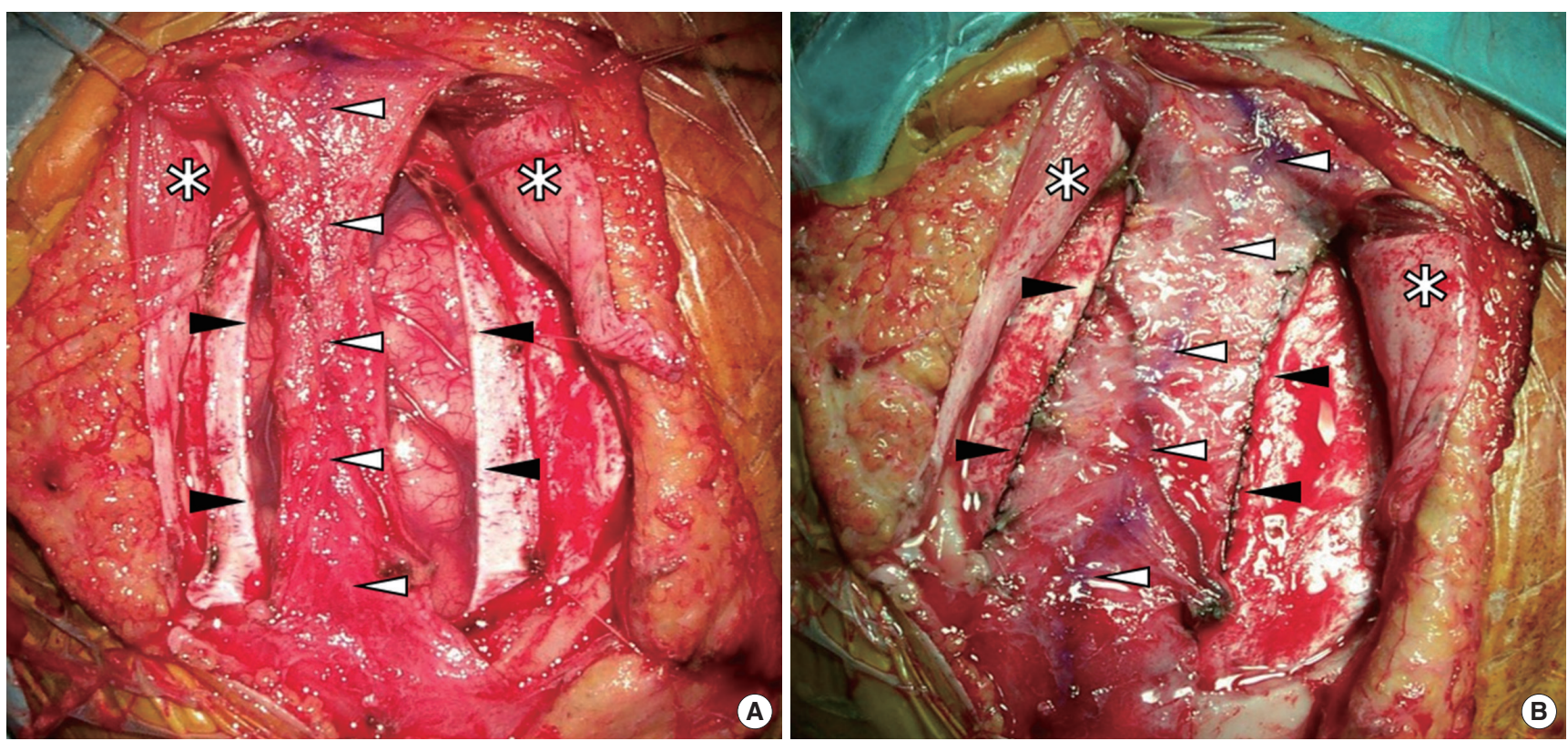

Figure 4. Intraoperative photos show the surgical procedure of encephalo-duro-arterio synangiosis (EDAS). (A) The formation of the arterial flap containing the parietal branch of the superficial temporal artery (STA) and surrounding galea tissue as a cuff shape. Under the flap, the cortex is exposed through the ovoid craniotomy and durotomy. (B) Completed suture with dural margin. The proximal and distal end of the STA is kept connected without cutting (white arrowheads = the parietal branch of the STA; black arrowheads = the dural margin being sutured with the cuff of flap water-tightly; asterisk=the temporalis muscle split vertically and reflected). 
periosteum are important sources for indirect revascularization in this area. After harvesting the galeo-periosteal flap, a craniotomy crossing the midline is made on the frontal cranium. The dura is incised leaving a base for the superior sagittal sinus, followed by folding the dura into the hemispheric fissure if possible. ${ }^{39}$ The galeo-periosteal flap is sutured with incised dura margins to cover the frontal brain.

Since the cortical branches of the PCA are usually smaller in caliber, the use of an indirect bypass for PCA territories has also been well described. ${ }^{43,44}$ The occipital artery is used as a supply vessel in this and other procedures that are similar to EDAS. Additional techniques include omental flap surgery and multiple burr hole surgery, which have also been introduced as either primary or rescue surgeries after failed revascularization. Despite relatively few reports, neovascularization with these methods has been reported to be comparable to other methods. $^{45-48}$

\section{Perioperative complications}

Various studies have reported complications after revascularization surgery for MMD. Postoperative stroke with permanent neurologic deficits developed in $1.6 \%-16.0 \% \%^{6,24,26-28}$ of patients and was more frequent in adults than in pediatric patients. ${ }^{49} \mathrm{In}$ addition, permanent neurologic deficits developed in $0.9 \%$ $8.0 \%$ of those with peri-operative ischemic stroke. ${ }^{6,24,26,27}$ However, the radiologic incidence of ischemic stroke was higher than symptomatic stroke. Funaki et al. reported that postoperative diffusion weighted imaging (DWI)-defined lesions were detected in 13 (9.3\%) out of 140 direct bypass operations for MMD, although only four lesions (2.9\%) could be classified as a permanent complication. However, the incidence of postoperative DWI-detected lesions in the unstable group was significant at $33.3 \%$ ( 8 of 24 )..$^{50}$ These peri-operative ischemic complications can be caused by several factors. However, the most important factor is the hemodynamic status of the patients. A patient with an advanced Suzuki stage with a lower CBF is prone to intraoperative stroke development. During surgery, maintaining euvolemic status and the appropriate blood pressure are important, along with appropriate anesthetic care. In addition, both hypotension and hypercapnia can aggravate hypoperfusion during surgery. Maintaining the level of hemoglobin is also essential in for oxygen delivery capacity. Thus, it is very important to communicate with the anesthesiologist while planning revascularization surgery.

Hemorrhagic stroke leading to permanent disability as a complication of revascularization for MMD developed in $0.7 \%-8.0 \%$ of patients in previous studies. ${ }^{6,24,26-28}$ Postoperative epidural hematoma (EDH) requiring surgical treatment developed in $4.8 \%$ of pediatric patients with $\mathrm{MMD}$, which was significantly higher than for non-MMD craniotomies $(0.8 \%$, $P<0.001) .{ }^{51}$ Controlling immediate postoperative stroke is the most important factor in securing the benefit of revascularization surgery for MMD, because most incidences of stroke develop in the immediate postoperative period. ${ }^{6}$

Another well-known phenomenon causing postoperative neurologic changes is hyperperfusion syndrome (21.5\%$50.0 \%){ }^{52-55}$ This phenomenon characteristically presents with transient neurologic deterioration with increased blood flow, and mostly develops in patients with MMD after surgery. ${ }^{55}$ Several possible mechanisms of hyperperfusion syndrome have been suggested. Due to a decreased vascular reserve capacity, the auto-regulatory function of the cerebral arteries in patients with MMD is severely impaired. After direct bypass, an increased amount of blood flows through these impaired arteries that cannot regulate the excessive flow. ${ }^{56}$ Moreover, due to chronic ischemia, vascular permeability is increased in vessels of patients with MMD. ${ }^{52}$ Although some reports introduced the idea of lowering systemic blood pressure as treatment for hyperperfusion syndrome, ${ }^{56}$ it can cause ischemic stroke because hyperperfusion is a local phenomenon associated with global hypoperfusion. Rather, adequate use of fluids and maintaining systemic blood pressure according to pre-operative levels are important for preventing further damage from ischemic or hemorrhagic stroke.

During surgery, the arteries of the scalp (i.e. STA and OA) are harvested and implanted into the intracranial space. This causes scalp ischemia that can lead to skin problems after revascularization. The incidence of scalp problems is reportedly $17.6 \%-21.4 \% .57,58$ Double-barrel procedures using both branches of the STA and a history of diabetes mellitus were found to be risk factors for wound-related complications. ${ }^{58}$ Moreover, a relationship between necrosis and smoking was noted. ${ }^{57} \mathrm{To}$ prevent skin necrosis after revascularization, the margin of the skin incision should be preserved without coagulation for hemostasis. During skin closure, meticulous inspection is required to limit any excessive tension of suture materials.

\section{Treatment outcomes}

The most important role of surgery is preventing further stroke for patients with MMD. Although it has been rarely reported, conservatively managed patients with MMD experienced stroke at a rate ranging between $3.2 \%-15.0 \%$ annually. ${ }^{15-18}$ In order to compare the stroke rate with the conservative group, reports regarding stroke development after surgical 
Table 1. Literature reporting post-operative stroke development after revascularization

\begin{tabular}{|c|c|c|c|c|c|}
\hline Authors & Year & Number of patients & Post-operative stroke & Follow-up Duration (month) & Annual stroke rate \\
\hline \multicolumn{6}{|c|}{ Direct revascularization for adults } \\
\hline Kuroda et al. ${ }^{67}$ & 2010 & 47 & 1 & 63 & 0.4 \\
\hline Bang et al. ${ }^{26}$ & 2012 & 61 & 1 & 64 & 0.3 \\
\hline Gross et al.$^{64}$ & 2013 & 29 & 2 & & 5.4 \\
\hline Cho et al. ${ }^{24}$ & 2014 & 60 & 3 & 71 & 0.8 \\
\hline Kim et al. ${ }^{6}$ & 2015 & 301 & 18 & 45 & 1.6 \\
\hline Arias et al..$^{59}$ & 2015 & 6 & 0 & 36 & 0.0 \\
\hline Sum & & 504 & 25 & & $1.4^{*}$ \\
\hline \multicolumn{6}{|c|}{ Direct revascularization for children } \\
\hline Karasawa et al. ${ }^{66}$ & 1992 & 104 & 2 & 115 & 0.2 \\
\hline Kuroda et al. ${ }^{67}$ & 2010 & 28 & 0 & 73 & 0.0 \\
\hline Funaki et al. ${ }^{62}$ & 2014 & 58 & 4 & 217 & 0.4 \\
\hline Sum & & 190 & 6 & & $0.2^{*}$ \\
\hline \multicolumn{6}{|c|}{ Indirect revascularization for adults } \\
\hline Bang et al. ${ }^{26}$ & 2012 & 14 & 2 & 64 & 2.7 \\
\hline Bao et al..$^{60}$ & 2012 & 470 & 60 & 27 & 5.8 \\
\hline Gross et al..$^{64}$ & 2013 & 13 & 5 & & 14.3 \\
\hline Imai et al. ${ }^{65}$ & 2015 & 36 & 1 & 72 & 0.5 \\
\hline Arias et al..$^{59}$ & 2015 & 9 & 0 & 36 & 0.0 \\
\hline Noh et al. ${ }^{18}$ & 2015 & 45 & 12 & 46 & 7.0 \\
\hline Sum & & 587 & 80 & & $5.6^{*}$ \\
\hline \multicolumn{6}{|c|}{ Indirect revascularization for children } \\
\hline Goda et al. ${ }^{63}$ & 2004 & 23 & 0 & 144 & 0.0 \\
\hline Scott et al. ${ }^{68}$ & 2004 & 126 & 15 & 61 & 2.3 \\
\hline Imai et al. ${ }^{65}$ & 2015 & 29 & 0 & 90 & 0.0 \\
\hline Bao et al. ${ }^{61}$ & 2015 & 288 & 20 & 52 & 1.6 \\
\hline Sum & & 466 & 35 & & $1.6^{*}$ \\
\hline
\end{tabular}

*The overall annual incidence was calculated with the weighted average.

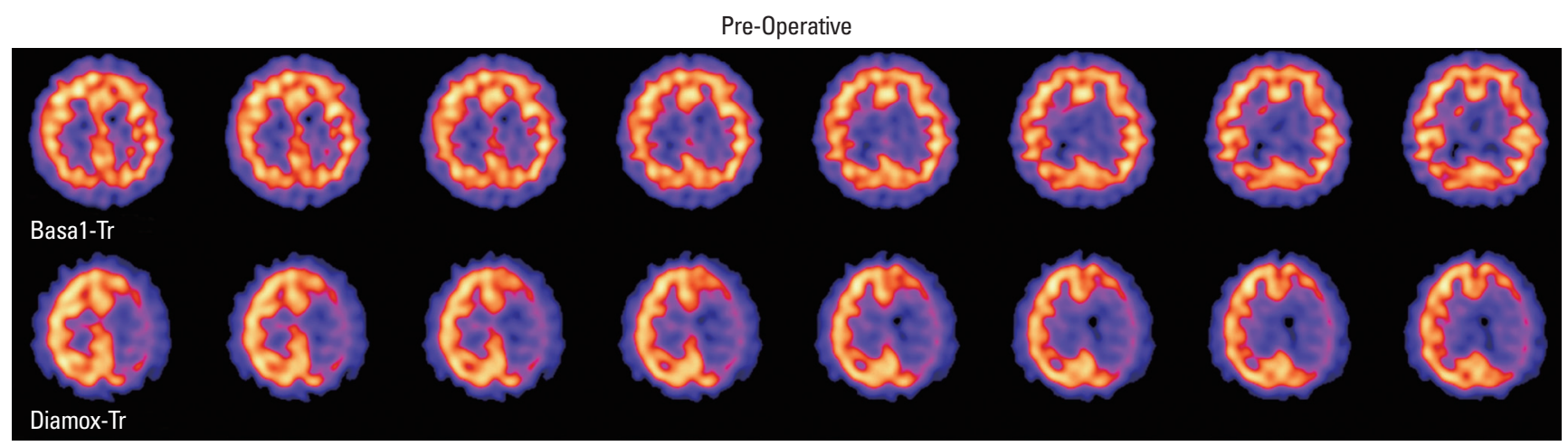

Post-Operative

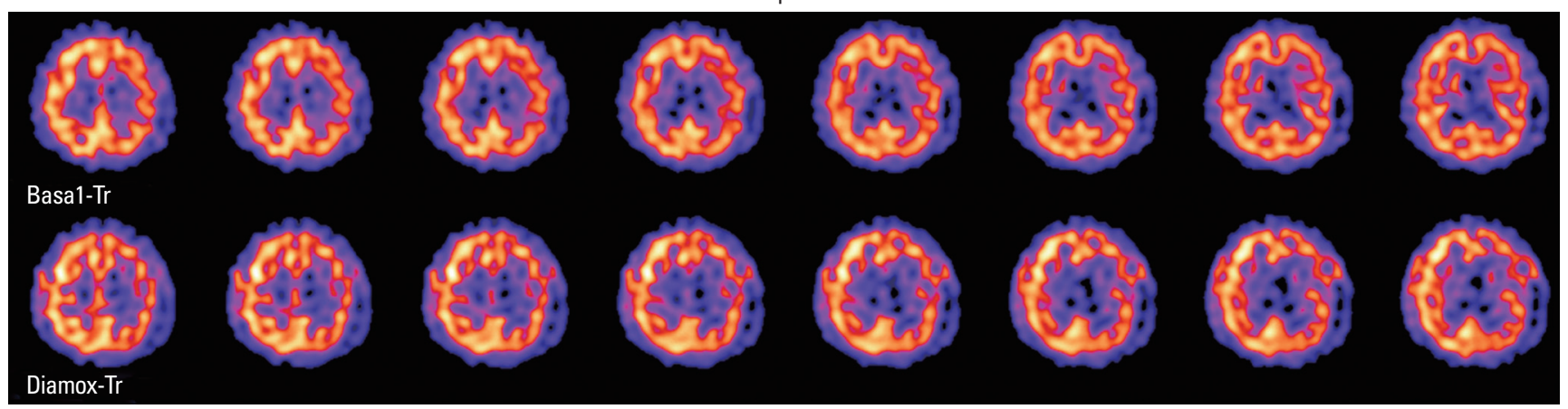

Figure 5. Post-operative single-photon emission computed tomography showing improved vascular reserve in the Diamox study comparing the pre-operative status. 
revascularization were reviewed. Among all studies, an annual incidence of post-operative stroke could be identified by surgical methods and age group (adult/pediatric) in 14 studies (Table 1). ${ }^{6,18,24,26,59-68}$

Direct or combined revascularization for adult patients with MMD seems to be effective for preventing stroke. ${ }^{6,24,26,59,64,67}$ Five studies with long term follow up ( $>36$ months) reported an annual stroke rate after direct revascularization of $0.0 \%-1.6 \%{ }^{6,24,26,59,67}$ Although Gross et al. reported a higher annual incidence (5.4\%), their results may be due to a shorter follow-up duration. Since most post-operative stroke develops in the peri-operative period, careful interpretation of these results is required. Among these six studies, the weighted average of the annual stroke rate was $1.4 \%$ in 504 adult patients who underwent direct revascularization, which is noticeably lower than the conservative treatment results. The relative risk reduction for cerebral infarction by direct revascularization was reportedly $70.7 \%{ }^{6}$

Direct revascularization for pediatric patients with MMD may also be effective in preventing further stroke. Over a sixyear follow-up period, six patients experienced post-operative stroke out of 190 pediatric patients who underwent direct revascularization. Especially, Kuroda et al. ${ }^{67}$ reported no stroke development during the 73 months following direct revascularization in pediatric patients with MMD. The weighted average of the annual stroke rate in three studies was $0.2 \% .62,66,67$ These favorable outcomes could be achieved by improving $\mathrm{CBF}$ and vascular reserve capacity (Figure 5). ${ }^{25,26,69}$

However, the incidence of newly developed cerebral infarction after indirect revascularization was reportedly somewhat higher than for the direct method. In reports examining adult cases treated with indirect revascularization, $0 \%-14.3 \%$ of patients annually experienced postoperative stroke. ${ }^{18,26,59,60,64,65}$ The weighted average of annual stroke was $5.6 \%$ among 587 patients. On the other hand, indirect revascularization for pediatric patients with MMD seemed to be more effective than for adult patients. Four studies with a relatively longer followup ( > 52 months) showed that the weighted average of annual stroke after indirect revascularization as $1.6 \%$ in pediatric cases. ${ }^{61,63,65,68}$ Thus, indirect revascularization seems to be more effective in pediatric patients with MMD than in adults. In addition, surgically treated pediatric patients with MMD showed a comparable rate of good social adaptation. ${ }^{70}$ Nevertheless, the effectiveness of surgery for long-term protection of cognitive function is unclear even if the initial surgical treatment may result in good neurological outcomes. ${ }^{71}$

While the effectiveness of revascularization for ischemic MMD has been generally accepted, surgical indications for MMD with a hemorrhagic presentation also remain undeter- mined. In the 1980s, indirect revascularization was considered to be more effective for prevention of further hemorrhage than direct revascularization. ${ }^{72}$ However, Aoki reported unsatisfactory results for prevention of recurrent hemorrhage using indirect revascularization. ${ }^{73}$ Nevertheless, since 2010, the effectiveness of surgical revascularization for hemorrhagic MMD has been reported. ${ }^{74}$ Moreover, indirect revascularization for hemorrhagic MMD has shown effectiveness in pediatric patients. ${ }^{75}$ Jiang et al. reported a hemorrhage recurrence rate of $1.9 \%$ at two-year follow-up after direct revascularization in adult patients. The rate of hemorrhagic stroke development was estimated as $11.5 \%$ with conservative management in previous studies. ${ }^{67,16,17}$ The level of evidence for prevention of further hemorrhage using direct revascularization became more robust in the Japan Adult Moyamoya Trial. ${ }^{7}$ However, this requires cautious interpretation because only marginal statistical significance was achieved by extensive bilateral direct revascularization.

\section{Conclusion}

Due to the progressive nature of the disease, surgical treatment for MMD should be considered for symptomatic patients. For pediatric patients, early diagnosis and active intervention before irreversible brain damage occurs are mandatory. Surgical revascularization is an effective treatment modality for preventing both ischemic and hemorrhagic stroke.

\section{References}

1. Suzuki J, Takaku A. Cerebrovascular "moyamoya” disease. Disease showing abnormal net-like vessels in base of brain. Arch Neurol 1969;20:288-299.

2. Fujimura M, Sonobe S, Nishijima Y, Niizuma K, Sakata H, Kure $S$, et al. Genetics and Biomarkers of Moyamoya Disease: significance of RNF213 as a Susceptibility Gene. J Stroke 2014;16: 65-72.

3. Kim T, Lee H, Bang JS, Kwon OK, Hwang G, Oh CW. Epidemiology of Moyamoya Disease in Korea: based on National Health Insurance Service Data. J Korean Neurosurg Soc 2015; 57:390-395.

4. Fukui M. Guidelines for the diagnosis and treatment of spontaneous occlusion of the circle of Willis ('moyamoya' disease). Research Committee on Spontaneous Occlusion of the Circle of Willis (Moyamoya Disease) of the Ministry of Health and Welfare, Japan. Clin Neurol Neurosurg 1997;99(Suppl 2):S238-240.

5. Narisawa A, Fujimura M, Tominaga T. Efficacy of the revascularization surgery for adult-onset moyamoya disease with the 
progression of cerebrovascular lesions. Clin Neurol Neurosurg 2009;111:123-126.

6. Kim T, Oh CW, Kwon OK, Hwang G, Kim JE, Kang HS, et al. Stroke prevention by direct revascularization for patients with adult-onset moyamoya disease presenting with ischemia. J Neurosurg 2015:1-6.

7. Miyamoto S, Yoshimoto T, Hashimoto N, Okada Y, Tsuji I, Tominaga T, et al. Effects of extracranial-intracranial bypass for patients with hemorrhagic moyamoya disease: results of the Japan Adult Moyamoya Trial. Stroke 2014;45:1415-1421.

8. Kim SH, Kwon OK, Jung CK, Kang HS, Oh CW, Han MH, et al. Endovascular treatment of ruptured aneurysms or pseudoaneurysms on the collateral vessels in patients with moyamoya disease. Neurosurgery 2009;65:1000-1004.

9. Kim T, Kwon OK, Oh CW, Bang JS, Hwang G, Lee YJ. Intracranial stenting using a drug-eluting stent for moyamoya disease involving supraclinoid ICA: a case report. Neurol Med Chir (Tokyo) 2014;54:136-138.

10. Kuroda S, Ishikawa T, Houkin K, Nanba R, Hokari M, Iwasaki $\mathrm{Y}$. Incidence and clinical features of disease progression in adult moyamoya disease. Stroke 2005;36:2148-2153.

11. Lee SC, Jeon JS, Kim JE, Chung YS, Ahn JH, Cho WS, et al. Contralateral progression and its risk factor in surgically treated unilateral adult moyamoya disease with a review of pertinent literature. Acta Neurochir (Wien) 2014;156:103-111.

12. Kim SJ, Heo KG, Shin HY, Bang OY, Kim GM, Chung CS, et al. Association of thyroid autoantibodies with moyamoya-type cerebrovascular disease: a prospective study. Stroke 2010;41: 173-176.

13. Im SH, Oh CW, Kwon OK, Kim JE, Han DH. Moyamoya disease associated with Graves disease: special considerations regarding clinical significance and management. J Neurosurg 2005; 102:1013-1017.

14. Mineharu Y, Takagi Y, Takahashi JC, Hashikata H, Liu W, Hitomi $\mathrm{T}$, et al. Rapid progression of unilateral moyamoya disease in a patient with a family history and an RNF213 risk variant. Cerebrovasc Dis 2013;36:155-157.

15. Kuroda S, Hashimoto N, Yoshimoto T, Iwasaki Y; Research Committee on Moyamoya Disease in Japan. Radiological findings, clinical course, and outcome in asymptomatic moyamoya disease: results of multicenter survey in Japan. Stroke 2007;38: 1430-1435.

16. Gross BA, Du R. The natural history of moyamoya in a North American adult cohort. J Clin Neurosci 2013;20:44-48.

17. Cho WS, Chung YS, Kim JE, Jeon JP, Son YJ, Bang JS, et al. The natural clinical course of hemodynamically stable adult moyamoya disease. J Neurosurg 2015;122:82-89.

18. Noh HJ, Kim SJ, Kim JS, Hong SC, Kim KH, Jun P, et al. Long term outcome and predictors of ischemic stroke recurrence in adult moyamoya disease. J Neurol Sci 2015;359:381-388.

19. Kraemer M, Berlit P, Diesner F, Khan N. What is the expert's option on antiplatelet therapy in moyamoya disease? Results of a worldwide Survey. Eur J Neurol 2012;19:163-167.

20. Yamada S, Oki K, Itoh Y, Kuroda S, Houkin K, Tominaga T, et al. Effects of Surgery and Antiplatelet Therapy in Ten-Year Follow-Up from the Registry Study of Research Committee on Moyamoya Disease in Japan. J Stroke Cerebrovasc Dis 2016;25: 340-349.

21. Wang KC, Phi JH, Lee JY, Kim SK, Cho BK. Indirect revascularization surgery for moyamoya disease in children and its special considerations. Korean J Pediatr 2012;55:408-413.

22. Kim SK, Cho BK, Phi JH, Lee JY, Chae JH, Kim KJ, et al. Pediatric moyamoya disease: An analysis of 410 consecutive cases. Ann Neurol 2010;68:92-101.

23. Karasawa J, Kikuchi H, Furuse S, Kawamura J, Sakaki T. Treatment of moyamoya disease with STA-MCA anastomosis. J Neurosurg 1978;49:679-688.

24. Cho WS, Kim JE, Kim CH, Ban SP, Kang HS, Son YJ, et al. Long-term outcomes after combined revascularization surgery in adult moyamoya disease. Stroke 2014;45:3025-3031.

25. Fujimura M, Tominaga T. Lessons learned from moyamoya disease: outcome of direct/indirect revascularization surgery for 150 affected hemispheres. Neurol Med Chir (Tokyo) 2012; 52:327-332.

26. Bang JS, Kwon OK, Kim JE, Kang HS, Park H, Cho SY, et al. Quantitative angiographic comparison with the OSIRIS program between the direct and indirect revascularization modalities in adult moyamoya disease. Neurosurgery 2012;70:625-632.

27. Guzman R, Lee M, Achrol A, Bell-Stephens T, Kelly M, Do $\mathrm{HM}$, et al. Clinical outcome after 450 revascularization procedures for moyamoya disease. Clinical article. J Neurosurg 2009; 111:927-935.

28. Mesiwala AH, Sviri G, Fatemi N, Britz GW, Newell DW. Longterm outcome of superficial temporal artery-middle cerebral artery bypass for patients with moyamoya disease in the US. Neurosurg Focus 2008;24:E15.

29. Miyamoto S, Akiyama Y, Nagata I, Karasawa J, Nozaki K, Hashimoto N, et al. Long-term outcome after STA-MCA anastomosis for moyamoya disease. Neurosurg Focus 1998;5:E5.

30. Hayashi T, Shirane R, Tominaga T. Additional surgery for postoperative ischemic symptoms in patients with moyamoya disease: the effectiveness of occipital artery-posterior cerebral artery bypass with an indirect procedure: technical case report. Neurosurgery 2009;64:E195-196.

31. Amin-Hanjani S, Du X, Mlinarevich N, Meglio G, Zhao M, Charbel FT. The cut flow index: an intraoperative predictor of 
the success of extracranial-intracranial bypass for occlusive cerebrovascular disease. Neurosurgery 2005;56:75-85.

32. Matsushima T, Fukui M, Kitamura K, Hasuo K, Kuwabara Y, Kurokawa T. Encephalo-duro-arterio-synangiosis in children with moyamoya disease. Acta Neurochir (Wien) 1990;104:96102.

33. Karasawa J, Kikuchi H, Furuse S, Sakaki T, Yoshida Y. A surgical treatment of "moyamoya" disease "encephalo-myo synangiosis”. Neurol Med Chir (Tokyo) 1977;17:29-37.

34. Matsushima Y. Encephalo-duro-arterio-synangiosis: surgical anatomy associated with procedure. No Shinkei Geka 1990;18: 989-999.

35. Kinugasa K, Mandai S, Kamata I, Sugiu K, Ohmoto T. Surgical treatment of moyamoya disease: operative technique for encephalo-duro-arterio-myo-synangiosis, its follow-up, clinical results, and angiograms. Neurosurgery 1993;32:527-531.

36. Irikura K, Miyasaka Y, Kurata A, Tanaka R, Yamada M, Kan S, et al. The effect of encephalo-myo-synangiosis on abnormal collateral vessels in childhood moyamoya disease. Neurol Res 2000;22:341-346.

37. Kawamoto H, Kiya K, Mizoue T, Ohbayashi N. A modified burr-hole method 'galeoduroencephalosynangiosis' in a young child with moyamoya disease. A preliminary report and surgical technique. Pediatr Neurosurg 2000;32:272-275.

38. Takeuchi S, Abe H, Ozawa T, Tanaka R. Surgical treatment for moyamoya disease. Surgical effect of encephalo-galeo-synangiosis on moyamoya disease. Surgery for Cerebral Strok 2000; 28:98-103.

39. Kim CY, Wang KC, Kim SK, Chung YN, Kim HS, Cho BK. Encephaloduroarteriosynangiosis with bifrontal encephalogaleo (periosteal) synangiosis in the pediatric moyamoya disease: the surgical technique and its outcomes. Childs Nerv Syst 2003; 19:316-324.

40. Kim SK, Wang KC, Kim IO, Lee DS, Cho BK. Combined encephaloduroarteriosynangiosis and bifrontal encephalogaleo (periosteal) synangiosis in pediatric moyamoya disease. $\mathrm{Neu}$ rosurgery 2002;50:88-96.

41. Park JH, Yang SY, Chung YN, Kim JE, Kim SK, Han DH, et al. Modified encephaloduroarteriosynangiosis with bifrontal encephalogaleoperiosteal synangiosis for the treatment of pediatric moyamoya disease. Technical note. J Neurosurg 2007;106: 237-242.

42. Song YS, Oh SW, Kim YK, Kim SK, Wang KC, Lee DS. Hemodynamic improvement of anterior cerebral artery territory perfusion induced by bifrontal encephalo(periosteal) synangiosis in pediatric patients with moyamoya disease: a study with brain perfusion SPECT. Ann Nucl Med 2012;26:47-57.

43. Kim DS, Kye DK, Cho KS, Song JU, Kang JK. Combined di- rect and indirect reconstructive vascular surgery on the frontoparieto-occipital region in moyamoya disease. Clin Neurol Neurosurg 1997;99(Suppl 2):S137-141.

44. Lee JY, Kim SK, Phi JH, Wang KC. Posterior Cerebral Artery Insufficiency in Pediatric Moyamoya Disease. J Korean Neurosurg Soc 2015;57:436-439.

45. Bruzoni M, Steinberg GK, Dutta S. Laparoscopic harvesting of omental pedicle flap for cerebral revascularization in children with moyamoya disease. J Pediatr Surg 2015:19.

46. Havlik RJ, Fried I, Chyatte D, Modlin IM. Encephalo-omental synangiosis in the management of moyamoya disease. Surgery 1992;111:156-162.

47. Zhao X, Wang C, Ji Y, Han C, Wang M. Therapeutic effect of multiple burr hole operation combined with dural inversion and periosteal synangiosis for moyamoya disease. $\mathrm{Br} J$ Neurosurg 2015:1-7.

48. Kawaguchi T, Fujita S, Hosoda K, Shose Y, Hamano S, Iwakura $\mathrm{M}$, et al. Multiple burr-hole operation for adult moyamoya disease. J Neurosurg 1996;84:468-476.

49. Kazumata K, Ito M, Tokairin K, Ito Y, Houkin K, Nakayama $\mathrm{N}$, et al. The frequency of postoperative stroke in moyamoya disease following combined revascularization: a single-university series and systematic review. J Neurosurg 2014;121:432440.

50. Funaki T, Takahashi JC, Takagi Y, Kikuchi T, Yoshida K, Mitsuhara $\mathrm{T}$, et al. Unstable moyamoya disease: clinical features and impact on perioperative ischemic complications. J Neurosurg 2015; 122:400-407.

51. Choi H, Lee JY, Phi JH, Kim SK, Cho BK, Wang KC. Postoperative epidural hematoma covering the galeal flap in pediatric patients with moyamoya disease: clinical manifestation, risk factors, and outcomes. J Neurosurg Pediatr 2013;12:181-186.

52. Fujimura M, Kaneta T, Mugikura S, Shimizu H, Tominaga T. Temporary neurologic deterioration due to cerebral hyperperfusion after superficial temporal artery-middle cerebral artery anastomosis in patients with adult-onset moyamoya disease. Surg Neurol 2007;67:273-282.

53. Fujimura M, Shimizu H, Inoue T, Mugikura S, Saito A, Tominaga T. Significance of focal cerebral hyperperfusion as a cause of transient neurologic deterioration after extracranial-intracranial bypass for moyamoya disease: comparative study with non-moyamoya patients using $\mathrm{N}$-isopropyl-p-[(123)I]iodoamphetamine single-photon emission computed tomography. Neurosurgery 2011;68:957-964.

54. Uchino H, Kuroda S, Hirata K, Shiga T, Houkin K, Tamaki N. Predictors and clinical features of postoperative hyperperfusion after surgical revascularization for moyamoya disease: a serial single photon emission $\mathrm{CT} /$ positron emission tomogra- 
phy study. Stroke 2012;43:2610-2616.

55. Kim JE, Oh CW, Kwon OK, Park SQ, Kim SE, Kim YK. Transient hyperperfusion after superficial temporal artery/middle cerebral artery bypass surgery as a possible cause of postoperative transient neurological deterioration. Cerebrovasc Dis 2008; 25:580-586.

56. Hayashi K, Horie N, Suyama K, Nagata I. Incidence and clinical features of symptomatic cerebral hyperperfusion syndrome after vascular reconstruction. World Neurosurg 2012;78:447454.

57. Katsuta T, Inoue T, Arakawa S, Uda K. Cutaneous necrosis after superficial temporal artery-to-middle cerebral artery anastomosis: is it predictable or avoidable? Neurosurgery 2001;49: 879-882.

58. Takanari K, Araki Y, Okamoto S, Sato H, Yagi S, Toriyama K, et al. Operative wound-related complications after cranial revascularization surgeries. J Neurosurg 2015:1-6.

59. Arias EJ, Dunn GP, Washington CW, Derdeyn CP, Chicoine MR, Grubb RL, Jr., et al. Surgical Revascularization in North American Adults with Moyamoya Phenomenon: Long-Term Angiographic Follow-up. J Stroke Cerebrovasc Dis 2015;24: 1597-1608.

60. Bao XY, Duan L, Li DS, Yang WZ, Sun WJ, Zhang ZS, et al. Clinical features, surgical treatment and long-term outcome in adult patients with Moyamoya disease in China. Cerebrovasc Dis 2012;34:305-313.

61. Bao XY, Duan L, Yang WZ, Li DS, Sun WJ, Zhang ZS, et al. Clinical features, surgical treatment, and long-term outcome in pediatric patients with moyamoya disease in China. Cerebrovasc Dis 2015;39:75-81.

62. Funaki T, Takahashi JC, Takagi Y, Yoshida K, Araki Y, Kikuchi $\mathrm{T}$, et al. Incidence of late cerebrovascular events after direct bypass among children with moyamoya disease: a descriptive longitudinal study at a single center. Acta Neurochir (Wien) 2014;156:551-559.

63. Goda M, Isono M, Ishii K, Kamida T, Abe T, Kobayashi H. Long-term effects of indirect bypass surgery on collateral vessel formation in pediatric moyamoya disease. J Neurosurg 2004;100:156-162.

64. Gross BA, Du R. Adult moyamoya after revascularization. Acta Neurochir (Wien) 2013;155:247-254.

65. Imai H, Miyawaki S, Ono H, Nakatomi H, Yoshimoto Y, Saito $\mathrm{N}$. The importance of encephalo-myo-synangiosis in surgical revascularization strategies for moyamoya disease in children and adults. World Neurosurg 2015;83:691-699.

66. Karasawa J, Touho H, Ohnishi H, Miyamoto S, Kikuchi H. Long-term follow-up study after extracranial-intracranial bypass surgery for anterior circulation ischemia in childhood moyamoya disease. J Neurosurg 1992;77:84-89.

67. Kuroda S, Houkin K, Ishikawa T, Nakayama N, Iwasaki Y. Novel bypass surgery for moyamoya disease using pericranial flap: its impacts on cerebral hemodynamics and long-term outcome. Neurosurgery 2010;66:1093-1101.

68. Scott RM, Smith JL, Robertson RL, Madsen JR, Soriano SG, Rockoff MA. Long-term outcome in children with moyamoya syndrome after cranial revascularization by pial synangiosis. $J$ Neurosurg 2004;100:142-149.

69. Han JS, Abou-Hamden A, Mandell DM, Poublanc J, Crawley $\mathrm{AP}$, Fisher JA, et al. Impact of extracranial-intracranial bypass on cerebrovascular reactivity and clinical outcome in patients with symptomatic moyamoya vasculopathy. Stroke 2011;42: 3047-3054.

70. Phi JH, Wang KC, Cho BK, Lee MS, Lee JH, Yu KS, et al. Long-term social outcome in children with moyamoya disease who have reached adulthood. J Neurosurg Pediatr 2011;8:303309.

71. Shim KW, Park EK, Kim JS, Kim DS. Cognitive Outcome of Pediatric Moyamoya Disease. J Korean Neurosurg Soc 2015;57: 440-444.

72. Nakagawa Y, Gotoh S, Shimoyama M, Ohtsuka K, Mabuchi S, Sawamura Y, et al. Reconstructive operation for moyamoya disease. Surgical indication for the hemorrhagic type, and preferable operative methods. Neurol Med Chir (Tokyo) 1983;23: 464-470.

73. Aoki N. Cerebrovascular bypass surgery for the treatment of Moyamoya disease: unsatisfactory outcome in the patients presenting with intracranial hemorrhage. Surg Neurol 1993;40: 372-377.

74. Liu X, Zhang D, Shuo W, Zhao Y, Wang R, Zhao J. Long term outcome after conservative and surgical treatment of haemorrhagic moyamoya disease. J Neurol Neurosurg Psychiatry 2013; 84:258-265.

75. Ahn JH, Wang KC, Phi JH, Lee JY, Cho BK, Kim IO, et al. Hemorrhagic moyamoya disease in children: clinical features and surgical outcome. Childs Nerv Syst 2012;28:237-245. 